\title{
Multi-Layer Interdigitated Power Distribution Networks
}

\author{
Renatas Jakushokas, Student Member, IEEE, and Eby G. Friedman, Fellow, IEEE
}

\begin{abstract}
Higher operating frequencies and greater power demands have increased the requirements on the power and ground network. Simultaneously, due to the larger current loads, current densities are increasing, making electromigration an important design issue. In this paper, methods for optimizing a multi-layer interdigitated power and ground network are presented. Based on the resistive and inductive (both self- and mutual) impedance, a closed-form solution for determining the optimal power and ground wire width is described, producing the minimum impedance for a single metal layer. Electromigration is considered, permitting the appropriate number of metal layers to be determined. The tradeoff between the network impedance and current density is investigated. Based on 65-, 45-, and 32-nm CMOS technologies, the optimal width as a function of metal layer is determined for different frequencies, suggesting important trends for interdigitated power and ground networks.
\end{abstract}

Index Terms-Current density, interdigitated structure, minimal impedance, power and ground (P/G) networks, width optimization.

\section{INTRODUCTION}

W ITH high operating frequencies and scaled geometries, the power and ground $(\mathrm{P} / \mathrm{G})$ distribution network requires greater design optimization to effectively provide higher current flow. These higher currents increase voltage losses within the $\mathrm{P} / \mathrm{G}$ network, while the power supply voltage is decreasing with advanced technologies, providing lower noise margins.

With flip-chip packaging, the package inductance is reduced [1], making the on-chip inductance more significant. Since voltage variations within a $\mathrm{P} / \mathrm{G}$ network are due to $I R$ [2], [3] and $L(d i) /(d t)$ [4] voltage drops, the effective resistance and inductance are the primary foci of the optimization process. At higher frequencies, the inductive impedance is dominant, requiring accurate estimation of the effective inductance.

An interdigitated $\mathrm{P} / \mathrm{G}$ distribution network structure where a few wide lines are replaced by a large number of narrow lines is often used to reduce the inductive effect [5], [6]. Different $\mathrm{P} / \mathrm{G}$ structures have been compared in [7], [8], where the interdigitated structure is shown to achieve the greatest reduction in inductance.

Manuscript received July 14, 2009; revised November 05, 2009 and January 19, 2010. First published March 29, 2010; current version published April 27, 2011. This work was supported in part by the National Science Foundation under Grant CCF-0541206, Grant CCF-0811317, and Grant CCF-0829915, by grants from the New York State Office of Science, Technology and Academic Research to the Center for Advanced Technology in Electronic Imaging Systems, and by grants from Intel Corporation, Eastman Kodak Company, and Freescale Semiconductor Corporation.

The authors are with the Electrical and Computer Engineering Department, University of Rochester, Rochester, NY 14627 USA (e-mail: jakushok@ece. rochester.edu; friedman@ece.rochester.edu).

Digital Object Identifier 10.1109/TVLSI.2010.2043453

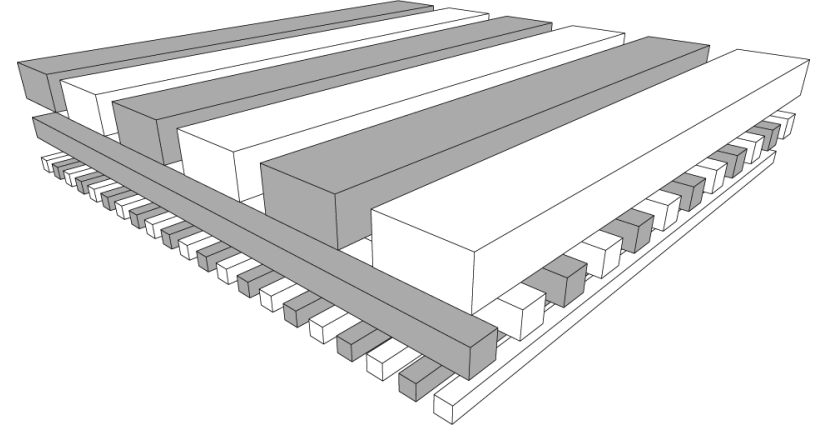

Fig. 1. Global interdigitated $\mathrm{P} / \mathrm{G}$ distribution structure. The darker and lighter lines represent, respectively, the power and ground lines.

An interdigitated P/G distribution structure is typically located on several metal layers. Each layer consists of interdigitated power and ground wires, where the direction of the wires is perpendicular to the direction of the wires in the previous layer, as depicted in Fig. 1. Routing flexibility and reduced inductance are two primary advantages of an interdigitated P/G distribution structure. With advancements in technology, additional metal layers are provided [9], permitting the dedication of several metal layers to the $\mathrm{P} / \mathrm{G}$ network. Due to electromigration, the maximum current is limited; therefore, a larger number of metal layers passes higher current to the system under the same electromigration constraint.

The need for efficient $\mathrm{P} / \mathrm{G}$ networks has been recognized, and several algorithms and techniques to optimize the P/G distribution network have been reported [10], [11]. A routing tool for standard cell circuits to efficiently supply and distribute power has been proposed in [12]. A typical high complexity IC however includes a variety of circuits, therefore, routing the supply network within a standard cell design flow can produce an ineffective network. To overcome this issue, several algorithms based on different optimization techniques have been developed [13], [14]; however, only the package inductance is considered [14], neglecting the on-chip inductance. An algorithm based on partitioning the P/G network into smaller sections is proposed in [15], where $I R$ voltage drops are considered. With more advanced packaging techniques (such as flip-chip), the on-chip inductive noise $(L(d i) /(d t))$ is also important [1], [16]. To consider on-chip inductance in $P / G$ networks, a technique to simplify a mesh model of the resistance-inductance-capacitance $(R L C) \mathrm{P} / \mathrm{G}$ network is proposed [17], assuming the loads are treated as identical current sources. The significance of the on-chip inductance within paired and interdigitated P/G network structures is described in [18], where the inductance is treated as a local effect. In [19], the inductance model considers the mutual inductance between close and distant $\mathrm{P} / \mathrm{G}$ wires in 

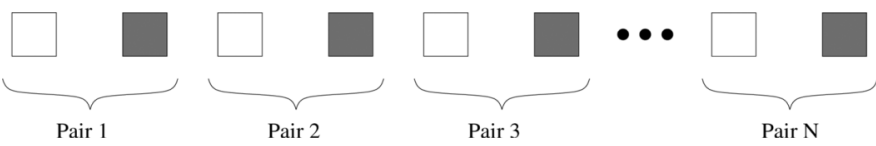

Fig. 2. $N$ pairs of a single layer within an interdigitated $\mathrm{P} / \mathrm{G}$ distribution structure.

interdigitated structures. Based on this model, a closed-form expression characterizing an interdigitated $\mathrm{P} / \mathrm{G}$ network structure is described, permitting the optimal width of a $\mathrm{P} / \mathrm{G}$ network that minimizes the network impedance to be determined. Based on the optimum width of the $\mathrm{P} / \mathrm{G}$ lines, a methodology to minimize the impedance under current density constraints for a multi-layer metal system is described in this paper.

This paper is organized as follows. The inductance of an interdigitated power and ground structure is discussed in Section II. A closed-form expression describing the minimum impedance for a single metal layer is presented in Section III. In Section IV, methods to lower the current density across multiple metal layers are discussed. Two different approaches are suggested. The tradeoff between the impedance of a $\mathrm{P} / \mathrm{G}$ network and the current density is presented in Section V. This paper is concluded in Section VI.

\section{INDUCTANCE OF INTERDIGITATED P/G DISTRIBUTION NETWORKS}

Estimating the inductance of a single layer within an interdigitated $\mathrm{P} / \mathrm{G}$ network structure is discussed in this section. A single layer of a network is depicted in Fig. 2, which consists of $N$ number of parallel power and ground wire pairs. The effective inductance of a single metal layer is

$$
\frac{1}{L_{\mathrm{eff}}}=\frac{1}{L_{1}}+\frac{1}{L_{2}}+\frac{1}{L_{3}}+\cdots+\frac{1}{L_{N}}
$$

where $L_{1}, L_{2}, L_{3}$, and $L_{N}$ are, respectively, the effective inductance of the first, second, third, and $N$ th pair of an interdigitated $\mathrm{P} / \mathrm{G}$ distribution network. Assuming the current flows in opposite directions in power and ground wires, the effective inductance of every pair can be determined based on [20]. In Fig. 3, the effective inductance normalized to the lowest effective inductance in a structure is depicted for each pair in a 100-pair interdigitated $\mathrm{P} / \mathrm{G}$ distribution network. The difference in inductance is small among all of the pairs, excluding those pairs closest to the boundary. The effect of the boundary is neglected, assuming each of the inductances is equal, permitting the effective inductance of a single layer within an interdigitated P/G distribution network structure to be determined [19]. A similar assumption is considered in [8], neglecting the mutual terms between the pairs, effectively treating the inductance as a local phenomena. By not neglecting distant mutual effects, the effective inductance can be estimated with higher precision. A derivation of the proposed effective inductance expression is presented in [19], based on the Wallis formula [21], resulting in

$$
L_{\mathrm{eff}}=\frac{\mu_{0} l}{N \pi}\left[\ln \left(\frac{w+s}{w+t}\right)+\frac{3}{2}+\ln \left(\frac{2}{\pi}\right)\right]
$$

where $N, \mu_{0}, l, w, t$, and $s$ are the number of power and ground pairs, permeability of the vacuum, length, width, and thickness of a single power or ground wire, and the spacing between the

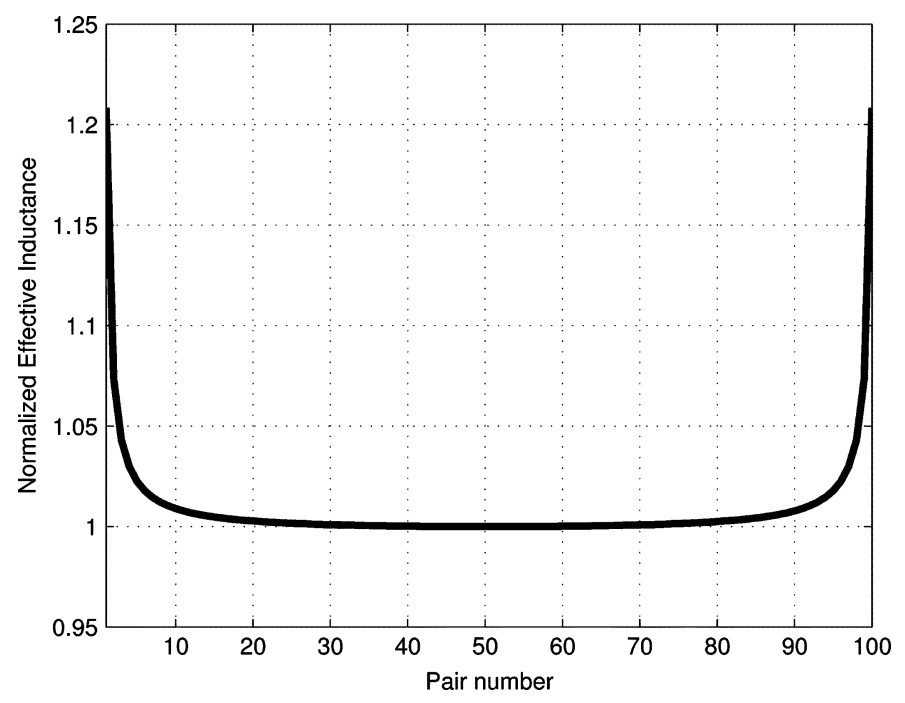

Fig. 3. Normalized effective inductance for each pair in a 100 pair interdigitated $\mathrm{P} / \mathrm{G}$ distribution network.

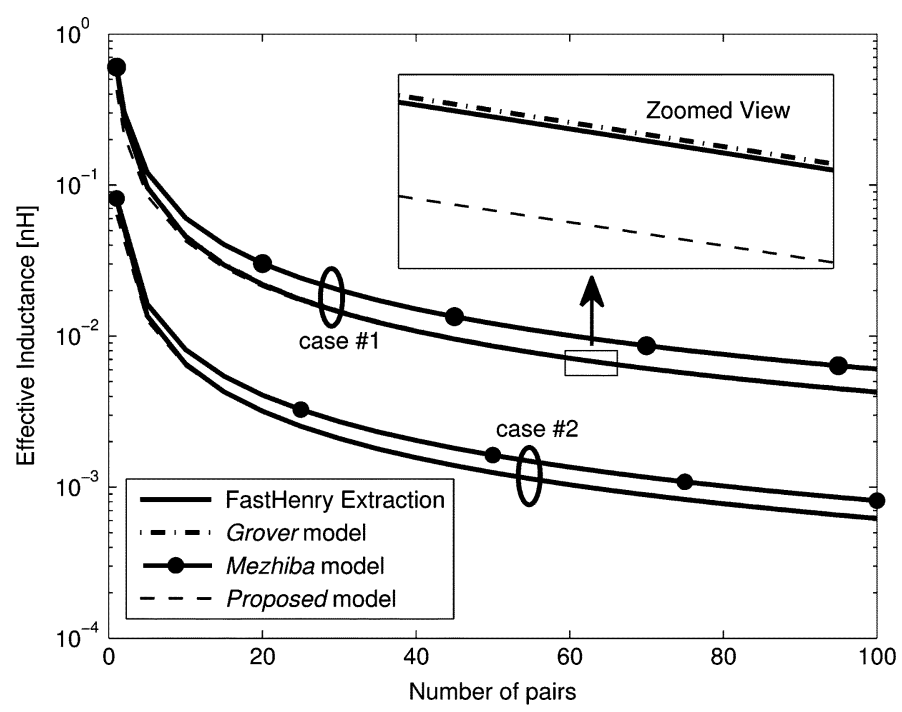

Fig. 4. Comparison of FastHenry, Grover, Mezhiba, and proposed models for two different design cases.

power and ground wires, respectively. The mutual inductance should be considered between all pairs, permitting the accuracy of the effective inductance to be improved by up to $30 \%$.

The proposed model, represented by (2), is compared among FastHenry [22], a multipole 3-D inductance extraction program, Grover, and Mezhiba models in Fig. 4. For the Grover model, the inductance of each pair is calculated individually and includes every mutual inductance component [20]. While the individual inductance of each pair is determined, the effective inductance of a single layer within an interdigitated $P / G$ network structure is estimated assuming the individual inductive lines are in parallel. Hence, the Grover model includes every mutual term of all of the wires in a system. In [8], the effective inductance is determined based on an approximation, where the inductance is treated as a local effect, and the mutual inductance between other pairs is neglected. This model is called the Mezhiba model. The effective inductance for the proposed model, represented by (2), is determined by placing a finite number $N$ of individual inductance pairs in parallel. The mutual 


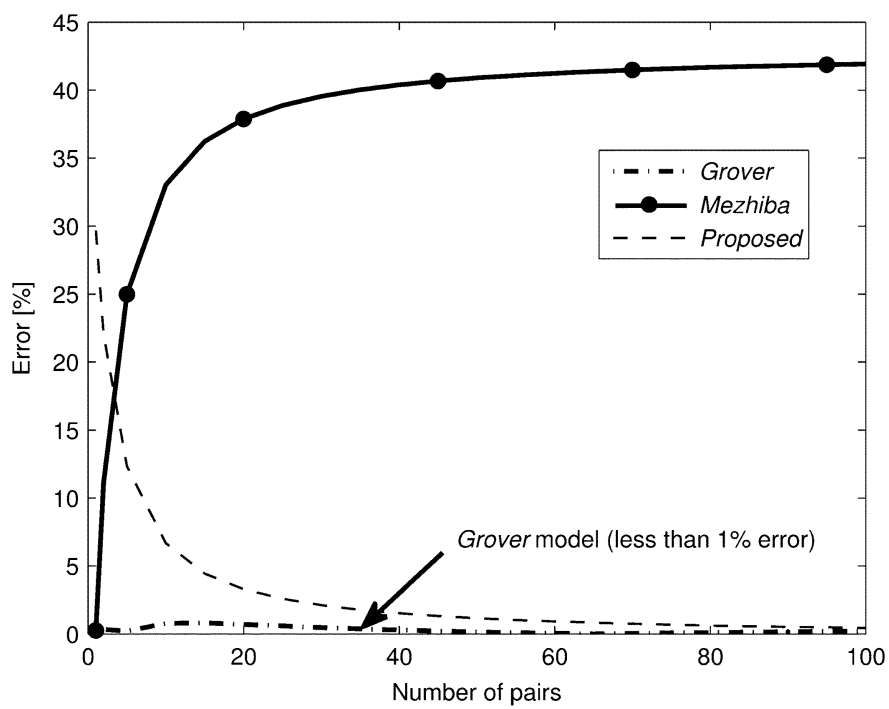

Fig. 5. Error comparison for the Grover, Mezhiba, and proposed models. All of the models are compared to FastHenry.

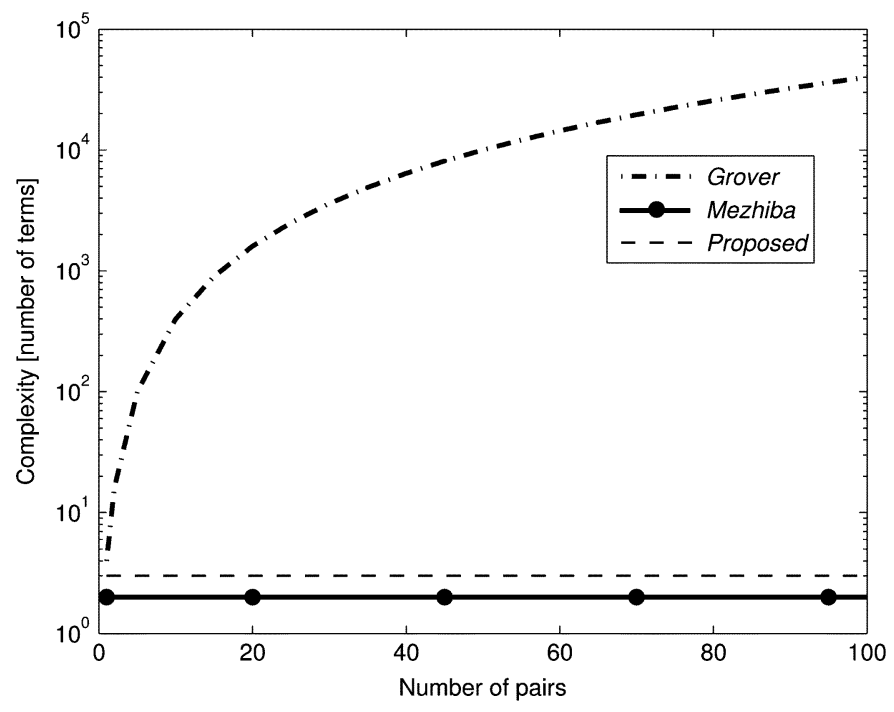

Fig. 6. Comparison of computational complexity among the Grover, Mezhiba, and proposed models.

inductance term of each $\mathrm{P} / \mathrm{G}$ pair is determined by assuming an infinite number of $\mathrm{P} / \mathrm{G}$ pairs on both sides of the specific pair. Since the magnitude of the mutual terms quickly declines to zero as a function of distance, this assumption is highly accurate in $\mathrm{P} / \mathrm{G}$ networks with a large number of interdigitated power and ground pairs.

The error of the Grover model is lowest; however, the computational complexity has polynomial increase with the higher number of wires in a $\mathrm{P} / \mathrm{G}$ distribution network. The error and complexity, presented in Figs. 5 and 6, are evaluated among the Grover, Mezhiba, and proposed models. The complexity of the proposed and Mezhiba models is independent of the number of $\mathrm{P} / \mathrm{G}$ pairs, while the proposed model converges to the values extracted by FastHenry, providing enhanced accuracy as compared to the Mezhiba model.

The area $A$ allocated for a $\mathrm{P} / \mathrm{G}$ network is typically constant

$$
A=l[N(w+s+w+s)]=2 l N(w+s)
$$

where the first $w+s$ term is the width and space of the power line, and the second $w+s$ term is for the ground line. Substituting (3) into (2), the effective inductance is

$$
L_{\mathrm{eff}}=\frac{2 l^{2} \mu_{0}(w+s)}{A \pi}\left[\ln \left(\frac{w+s}{w+t}\right)+\frac{3}{2}+\ln \left(\frac{2}{\pi}\right)\right] .
$$

Note that (4) considers both the self- and mutual inductance, where the mutual inductance is between an infinite number of pairs. This expression requires low computational time while providing high accuracy when estimating the inductance of an interdigitated power and ground distribution network.

\section{Single Metal LAYer CharaCteristics}

The following section is divided into subsections. The optimal line width for a single interdigitated metal layer is determined in Section III-A. The accuracy of the optimal line width and related issues are discussed in Section III-B.

\section{A. Optimal Width for Minimum Impedance}

An interdigitated $\mathrm{P} / \mathrm{G}$ distribution network is typically allocated over an entire upper metal layer, where the network is designed for lowest impedance. The resistance of a single power and ground pair is

$$
R=\rho \frac{2 l}{t w}
$$

where $\rho$ is the metal resistivity. $N$ pairs of power and ground wires are in parallel; the effective resistance of the network is therefore

$$
R_{\mathrm{eff}}=\frac{1}{N} \rho \frac{2 l}{t w} .
$$

Substituting (3) into (6), the effective resistance for constant area is

$$
R_{\mathrm{eff}}=\frac{4 l^{2} \rho(w+s)}{A t w} .
$$

For constant area, according to (7), wider power and ground wires reduce the effective resistance. With multiple thin lines, a large area is consumed by the line-to-line spacing, increasing the effective resistance of the network. The inductance under a constant area constraint has the opposite effect since the mutual inductance is dominant in an interdigitated $\mathrm{P} / \mathrm{G}$ distribution structure. A greater number of lines increases the mutual inductance, reducing the effective inductance, as described by (4).

The value of the effective impedance as a function of width (or number of pairs) is

$$
Z_{\mathrm{eff}}(w)=R_{\mathrm{eff}}(w)+j 2 \pi f L_{\mathrm{eff}}(w)
$$

where $f$ is the target frequency. At $5 \mathrm{GHz}$, (4) and (7) are compared to FastHenry, as shown in Fig. 7. A constant area of $1 \mathrm{~mm} \times 1 \mathrm{~mm}$ at the top metal layer for a 65-nm CMOS technology [23] is assumed. 


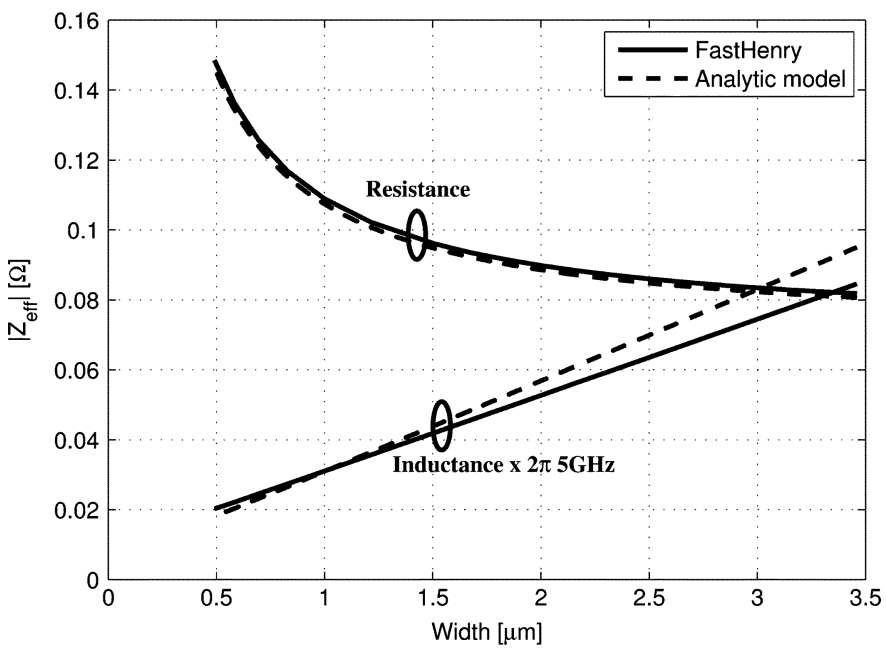

Fig. 7. Effective resistance and inductance at $5 \mathrm{GHz}$ as a function of width for a single layer within an interdigitated $\mathrm{P} / \mathrm{G}$ distribution network. The overall area is maintained constant.

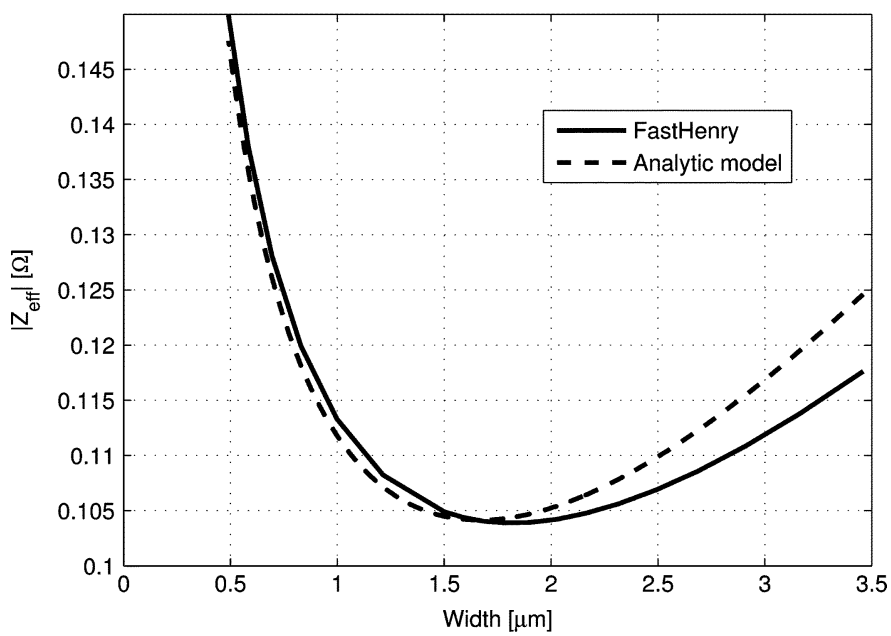

Fig. 8. Magnitude of the impedance of (9) and FastHenry.

Since the effect of the resistive and inductive impedance behaves inversely with increasing width, the objective is to minimize the overall impedance at a specific frequency. The absolute value of the effective impedance as a function of line width is

$$
\left|Z_{\mathrm{eff}}(w)\right|=\sqrt{R_{\mathrm{eff}}^{2}(w)+4 \pi^{2} f^{2} L_{\mathrm{eff}}^{2}(w)}
$$

as illustrated in Fig. 8.

Since the effective inductance in (4) is a transcendental function of width, a closed-form analytic solution can not be determined for the wire width that minimizes the impedance. A closed-form expression can, however, be determined for the special case where the line-to-line spacing is equal to the thickness of the metal in the effective inductance equation, resulting in

$$
w_{\mathrm{opt}}^{(0)} \approx \sqrt[3]{0.91 \frac{s \rho^{2}}{\mu_{o}^{2} t^{2} f^{2}}} .
$$

A detailed derivation of (10) is presented in Appendix A. A numerical solution based on $n$ iterations of the Newton-Raphson

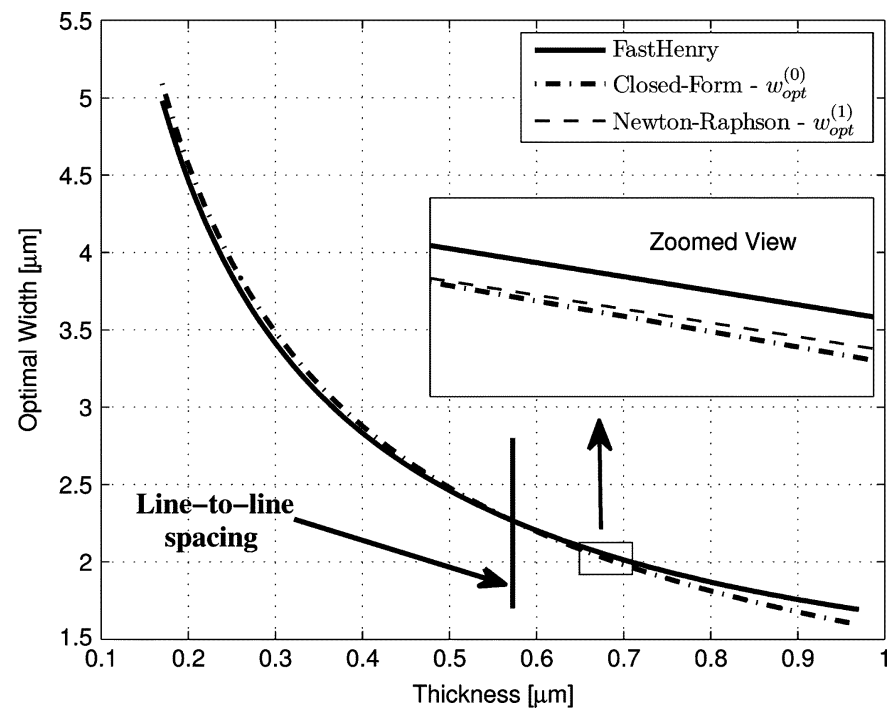

Fig. 9. Closed-form $w_{\mathrm{opt}}^{(0)}$ and $w_{\mathrm{opt}}^{(1)}$ based on the first iteration of the Newton-Raphson method as compared with FastHenry for different thicknesses.

method is used to determine the optimal width for all other spacings

$$
w_{\mathrm{opt}}^{(n)}=w_{\mathrm{opt}}^{(n-1)}-\frac{F^{\prime}\left(w_{\mathrm{opt}}^{(n-1)}\right)}{F^{\prime \prime}\left(w_{\mathrm{opt}}^{(n-1)}\right)}
$$

where $F \equiv\left|Z_{\mathrm{eff}}(w)\right|$ and $w_{\mathrm{opt}}^{(n-1)}$ is the $(n-1)^{\mathrm{st}}$ estimate of the optimal wire width. The initial estimate is based on (10). The number of iterations can be increased to enhance the accuracy of the optimal width.

Considering the resistance and inductance (both self- and mutual) of a network, (10) combined with (11) can be used to determine the optimal line width of an interdigitated $\mathrm{P} / \mathrm{G}$ network. The optimal line width produces the minimum impedance network at a target frequency.

\section{B. Optimal Width Characteristics}

A comparison among FastHenry, (10), and $w_{\mathrm{opt}}^{(1)}$ based on the first iteration of the Newton-Raphson method is shown in Fig. 9 for several different metal thicknesses. The spacing is chosen as the mid point between the thinnest and thickest metal layers for a 65-nm CMOS technology. A $5 \mathrm{GHz}$ frequency is assumed. The error between FastHenry and (10) reaches 6\%, while the error between FastHenry and $w_{\mathrm{opt}}^{(1)}$ is below $1 \%$. For those cases where the target accuracy is below the error of the initial estimate, the closed-form expression of $w_{\mathrm{opt}}^{(0)}$ is computationally efficient in determining the $\mathrm{P} / \mathrm{G}$ line width. If higher accuracy is required, the interdigitated $\mathrm{P} / \mathrm{G}$ wire width can be determined according to (11).

A comparison among FastHenry, (10), and $w_{\mathrm{opt}}^{(1)}$ based on the first iteration of the Newton-Raphson method is shown in Fig. 10 for different spacings. The spacing ranges from 0.54 $\mu \mathrm{m}$ (the lowest permitted spacing) to $15 \mu \mathrm{m}$. When the spacing is equal to the thickness, $w_{\mathrm{opt}}^{(0)}$ and $w_{\mathrm{opt}}^{(1)}$ are equal since the logarithmic term of (4) is zero. At spacings below $7 \mu \mathrm{m}$, (10) exhibits an error below 9\% as compared to FastHenry. For spacings up to $15 \mu \mathrm{m}$, the error between FastHenry and (10) 


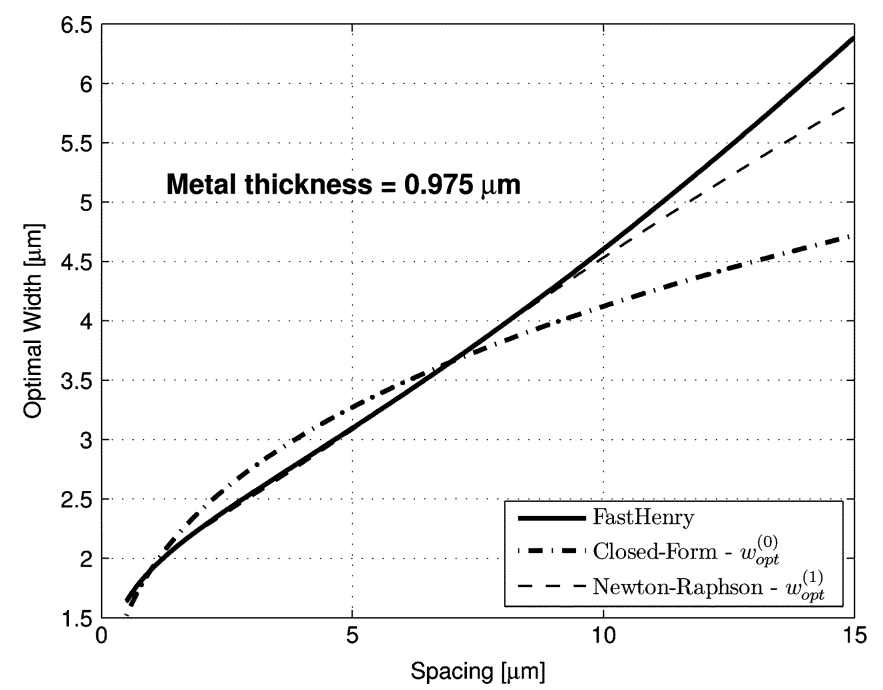

Fig. 10. Closed-form $w_{\mathrm{opt}}^{(0)}$ and $w_{\mathrm{opt}}^{(1)}$ based on the first iteration of the Newton-Raphson method as compared with FastHenry for different spacings.

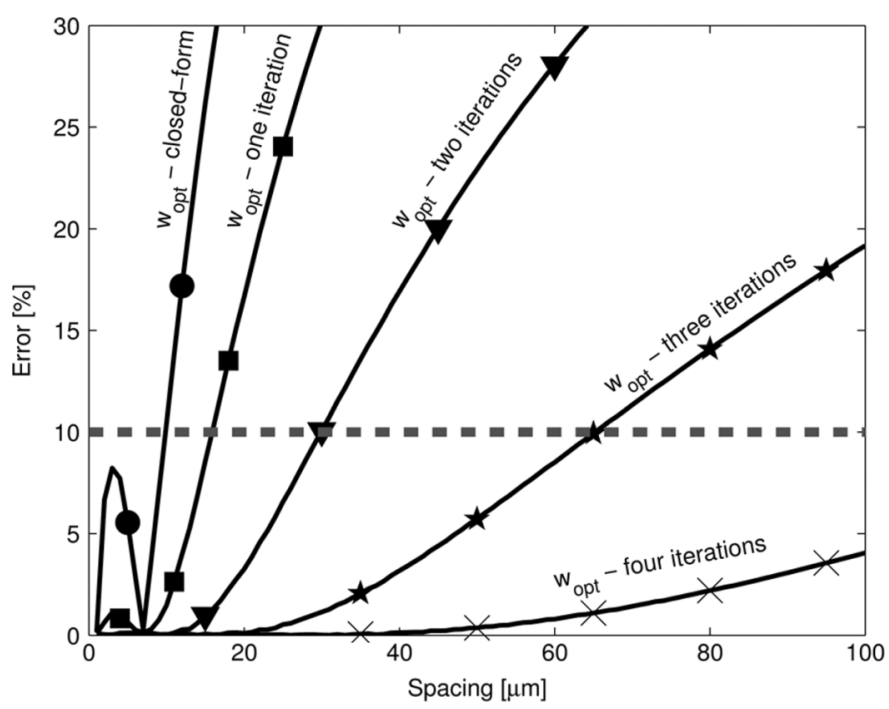

Fig. 11. Error of $w_{\text {opt }}$ is evaluated for several spacings using closed-form and one to four iterations of the Newton-Raphson method. The error is relative to FastHenry.

reaches $26 \%$, while the error with only the first iteration of the Newton-Raphson method is below $9 \%$. For spacings greater than $15 \mu \mathrm{m}$, additional iterations of the Newton-Raphson method are necessary.

The different number of iterations to determine $w_{\text {opt }}$ are evaluated in Fig. 11. The error is relative to FastHenry. Note that (10) assumes the spacing and thickness are equal. The closed-form expression is therefore only accurate for small spacings. For wider spacings, the Newton-Raphson method is preferred to accurately determine the optimal width. A larger number of iterations is needed for wider spacings since the error decreases significantly for large number of iterations. Spacings up to $100 \mu \mathrm{m}$ are evaluated, suggesting that four iterations are sufficient to determine the optimal width within $10 \%$ accuracy as compared to FastHenry.

Since the width is optimized for a target frequency, the effect on the frequency range of interest (from DC to the target frequency) is important. In the following discussion, a target frequency of $5 \mathrm{GHz}$ is assumed. In Fig. 12, the impedance of the network as a function of frequency is depicted. Three values of

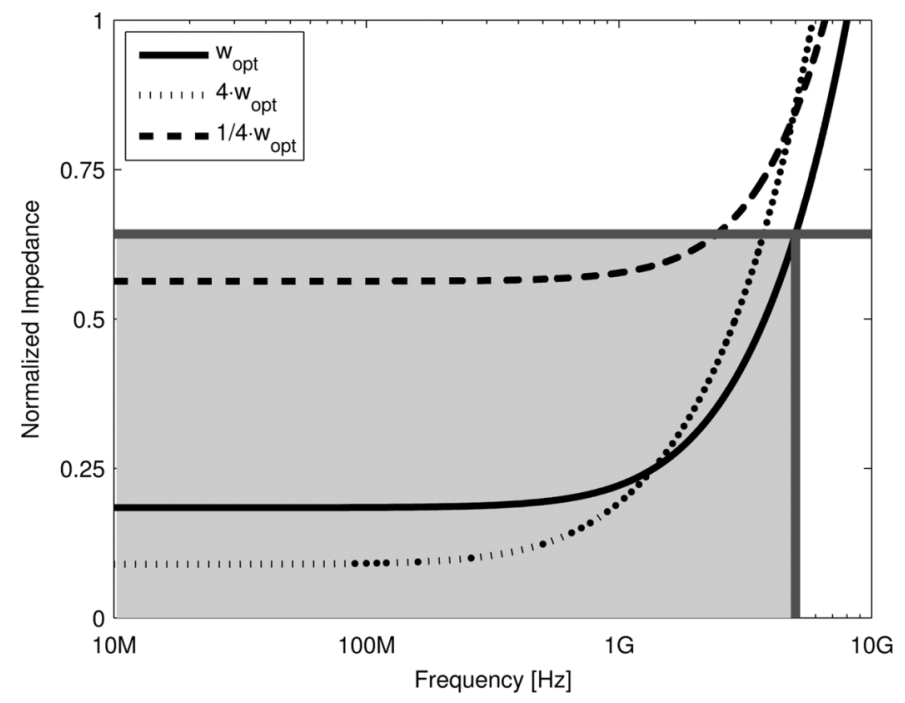

Fig. 12. Impedance over the frequency range of interest. Three different $P / G$ network line widths are depicted. The impedance is minimum at the target frequency with the optimum width.

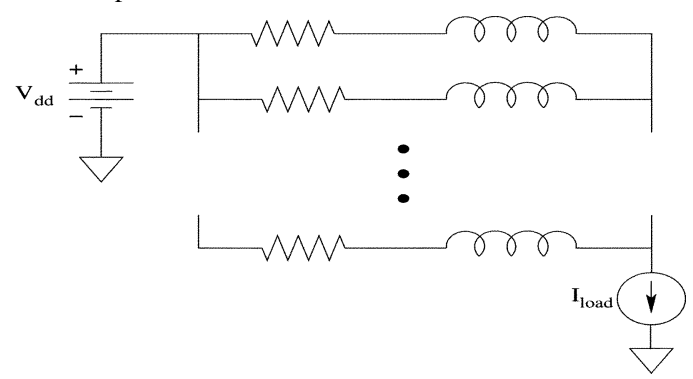

Fig. 13. Multi-layer P/G distribution network model. Each resistance and inductance represent, respectively, the effective resistance and inductance of a single layer within a P/G network.

the width are evaluated - the optimal width, a width four times greater than the optimal width, and a width four times smaller than the optimal width. Note that the area is maintained constant. An increasing width corresponds to a fewer number of interdigitated pairs within the P/G network (a thinner line corresponds to a higher number of interdigitated pairs).

As illustrated in Fig. 12, the minimum impedance at $5 \mathrm{GHz}$ is achieved using the optimal width, while a lower and higher line width increases, respectively, the resistive and inductive component of the overall impedance. At low frequencies, the $\mathrm{P} / \mathrm{G}$ network with wider lines produces a lower impedance, although more than the required impedance at the target frequency. In the example shown in Fig. 12, the network impedance with wider lines is below the target impedance only below $3.7 \mathrm{GHz}$. As depicted in Fig. 12, the P/G network with a smaller width satisfies the impedance requirements only up to $2.5 \mathrm{GHz}$.

\section{Multi-LAYER OptimizATiON}

Multi-layer systems can be approximated by the network shown in Fig. 13, where the resistance and inductance is, respectively, the effective resistance and inductance of a single layer within a $\mathrm{P} / \mathrm{G}$ distribution network [8]. This model treats the system as worse case since all of the current is assumed to flow through the entire layer. Electromigration is considered when optimizing a multi-layer system. 

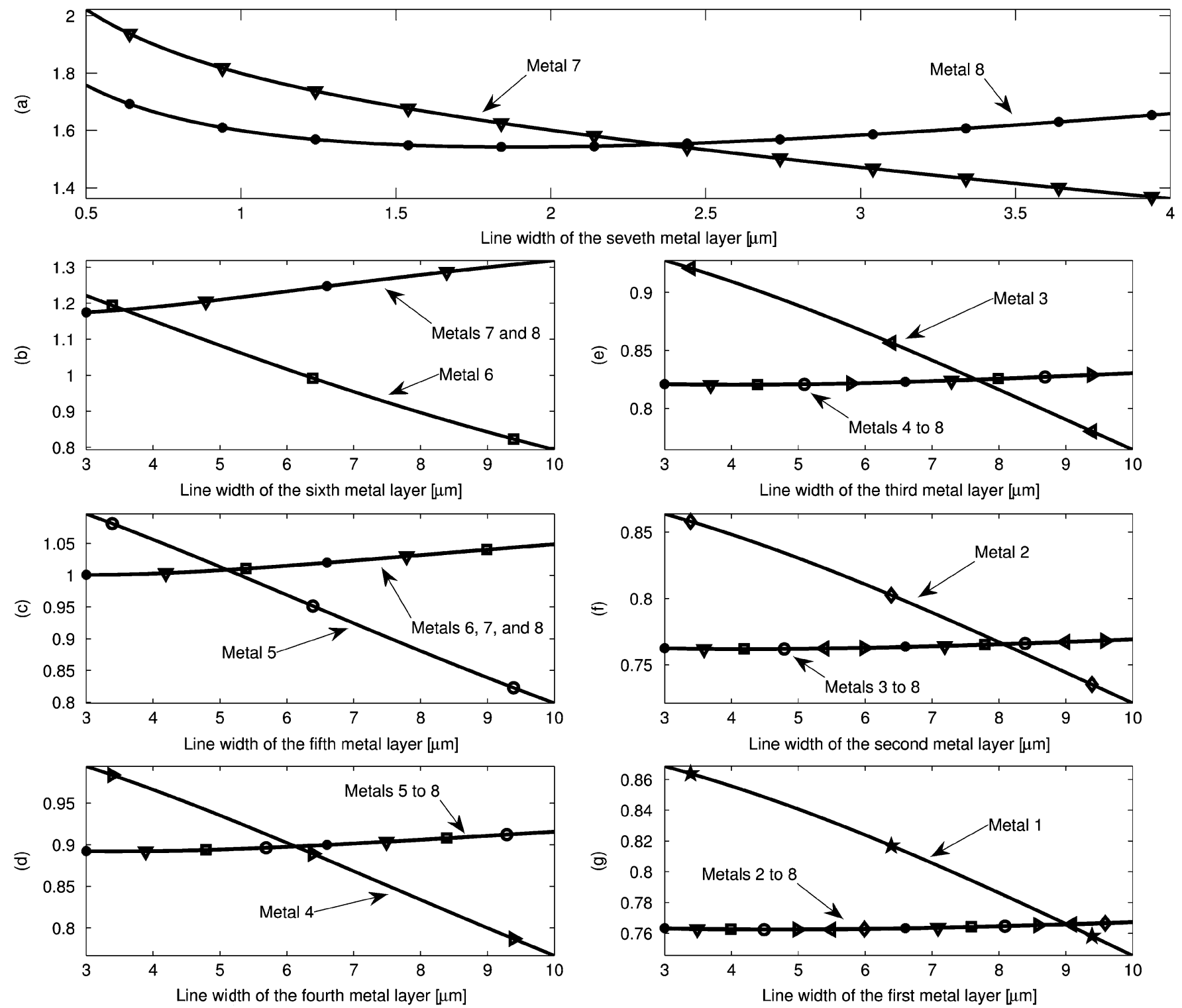

Fig. 14. Current density for multiple metal layers. The current density of the (a) seventh and eighth, (b) sixth, seventh, and eighth, (c) fifth, sixth, seventh, and eighth, (d) fourth to eighth, (e) third to eighth, (f) second to eighth, and (g) first to eighth metal layers. The width is determined at the intersection of the current density of the multiple metal layers. The $y$-axis for each figure is the current density in units of $\mathrm{mA} / \mu \mathrm{m}^{2}$, while the total current is assumed to be $1 \mathrm{~A}$.

The current density $C D$ of an arbitrary layer $m$ is

$$
C D_{m}=\frac{\left|i_{m}\right|}{\operatorname{CroSec}_{m}}
$$

where $i_{m}$ and $\mathrm{CroSec}_{m}$ are the current and cross section of layer $m$, respectively. The skin effect is considered in determining the cross-section of the layer,

$\operatorname{CroSec}_{m}= \begin{cases}N_{m} w_{m} t_{m}, & 2 \delta>w \\ N_{m} w_{m} t_{m}, & 2 \delta>t \\ 2 \delta\left[N_{m}\left(w_{m}+t_{m}\right)-2 \delta\right], & \text { otherwise }\end{cases}$

where $\delta$ is the skin depth. The skin depth is defined as

$$
\delta \equiv \sqrt{\frac{1}{\pi f \mu_{0} \sigma}}
$$

where $\sigma$ is the conductivity of the material.
Allocating additional metal layers for the power and ground distribution network distributes the overall current among a larger number of metal layers. The current density of a particular metal layer is therefore lower.

Two different approaches are considered for optimizing a multi-layer P/G network. In the first approach, the current density per layer is maintained equal for all of the layers, while providing a low P/G network impedance. The second approach minimizes the impedance, while considering electromigration. A tradeoff exists between the current density and the impedance of a $\mathrm{P} / \mathrm{G}$ distribution network. A lower impedance reduces the voltage drop, providing a higher noise margin.

\section{A. First Approach-Equal Current Density}

The first optimization approach for an interdigitated P/G distribution network structure is discussed in this subsection. The limiting current density is the highest current density among the 
layers. In this approach, the current density among the layers is maintained equal, minimizing the limiting current density of a $\mathrm{P} / \mathrm{G}$ network. A lower limiting current density enhances the reliability of a multi-layer system. The current flowing through an arbitrary layer $m$ is

$$
\left|i_{m}\right|=\frac{V_{\text {drop }}}{\left|Z_{m}\right|}
$$

where $V_{\text {drop }}$ and $Z_{m}$ are the voltage across the entire P/G distribution network and the impedance of the $m$ th layer, respectively. Substituting (17) into (12), the current density of the $m$ th metal layer is

$$
C D_{m}=\frac{V_{\mathrm{drop}}}{\left|Z_{m}\right|} \frac{1}{\mathrm{CroSec}_{m}} .
$$

Two layers, $m$ and $n$, provide the same current density when

$$
\left|Z_{m}\right| \operatorname{CroSec}_{m}=\left|Z_{n}\right| \mathrm{CroSec}_{n} .
$$

While the width of a single metal layer is optimized for minimum impedance, the width of the remaining metal layers is chosen to maintain equal current density, as described by (19). Pseudo-code for determining the width of the individual metal layers within a multi-layer system based on maintaining equal current density among the metal layers is provided in Appendix B.

Based on the proposed methodology, an eight layer P/G distribution network is described for a 65-nm CMOS technology. To evaluate the proposed methodology, all of the metal layers are available for the $\mathrm{P} / \mathrm{G}$ distribution, although in practical cases some metal layers are used for the signals, clock network, and shield lines.

Based on a $65-\mathrm{nm}$ CMOS technology, a width of $1.66 \mu \mathrm{m}$ is initially determined from (11) for the top (eighth) metal layer to minimize the impedance of a single metal layer. The width of the additional metal layers is based on maintaining equal current density according to (19). The current density per multiple metal layers is depicted in Fig. 14. Increasing the width of the lower metal layer affects the current density in the lower layer as well as the upper metal layer. Increasing the width of the lower metal layer changes the impedance of the lower metal layer (decreasing the resistance and increasing the inductance). The current is distributed among the different metal layers based on the relative impedance of the metal layers, resulting in larger current in the metal layer with lower impedance while changing the current density in all of the metal layers.

The width is determined at the intersection of the current density of multiple metal layers. The intersection is the width where equal current density among the multiple metal layers is maintained, lowering the limiting current density. As inferred in Fig. 14, this intersection occurs at a greater width for the lower metal layers, since the metal layers are thinner. This structure is therefore called the pyramid structure. The spacing, thickness, width, and number of interdigitated pairs for each metal layer in an eight layer P/G system is summarized in Table I.

The normalized current density is shown in Fig. 15 for an eight metal layer $\mathrm{P} / \mathrm{G}$ network based on the equal current density methodology. While the maximum current density for a specific
TABLE I

SPACING, THICKNESS, WIDTH, AND NUMBER OF INTERDIGITATED PAIRS FOR AN Eight Metal Layer System. The Eighth Metal Layer Is the Top Metal LAYER. SinCE THE LINES ARE WIDER, THE NUMBER OF INTERDIGITATED PAIRS IS LOWER FOR A CONSTANT AREA

\begin{tabular}{|c||c|c|c|c|}
\hline $\begin{array}{c}\text { Metal } \\
\text { Layer }\end{array}$ & $\begin{array}{c}\text { Thickness } \\
{[\mu \mathrm{m}]}\end{array}$ & $\begin{array}{c}\text { Spacing } \\
{[\mu \mathrm{m}]}\end{array}$ & $\begin{array}{c}\text { Width } \\
{[\mu \mathrm{m}]}\end{array}$ & $\begin{array}{c}\text { Number } \\
\text { of pairs }\end{array}$ \\
\hline \hline 8 & 0.975 & 0.540 & 1.66 & 227 \\
7 & 0.650 & 0.360 & 2.36 & 183 \\
6 & 0.430 & 0.240 & 3.56 & 131 \\
5 & 0.300 & 0.165 & 5.11 & 94 \\
4 & 0.250 & 0.140 & 6.13 & 79 \\
3 & 0.200 & 0.110 & 7.67 & 64 \\
2 & 0.190 & 0.105 & 8.07 & 61 \\
1 & 0.170 & 0.105 & 9.02 & 54 \\
\hline
\end{tabular}

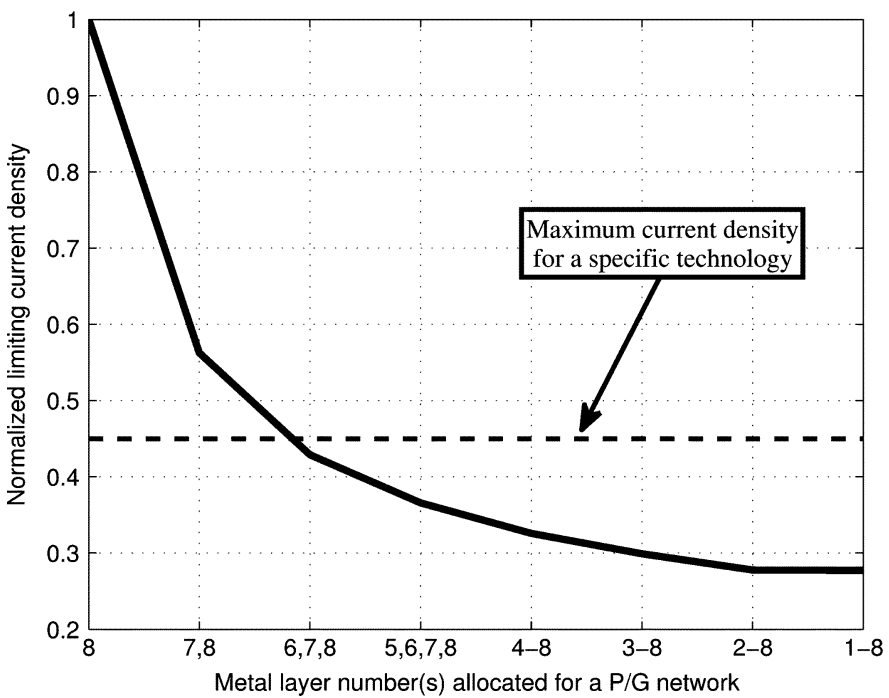

Fig. 15. Normalized limiting current density for different metal layers. The $x$-axis represents the metal layer number(s) allocated for a P/G network. The current density is highest when allocating only a single metal layer (layer number eight) for a $\mathrm{P} / \mathrm{G}$ network. The current density is reduced as additional metal layers are added.

technology, physical area, and current is known, the required number of metal layers for an interdigitated $\mathrm{P} / \mathrm{G}$ distribution network can be determined, as illustrated in Fig. 15.

Two additional $\mathrm{P} / \mathrm{G}$ network structures are compared with the proposed pyramid structure. These three structures are illustrated in Fig. 16. The width of the individual metal layers for the pyramid structure is listed in Table I. This structure is shown in Fig. 16(a). Note in the pyramid structure, the power and ground lines in the lower metal layers are wider. In conventional metal systems, the power and ground lines are wider at the higher metal layers, as illustrated in Fig. 16(b). For this structure, the width of the metal layers is the opposite of the pyramid structure, and is therefore called the inverted pyramid (standard) structure. In Fig. 16(c), the width of each metal layer is maintained constant at $5.5 \mu \mathrm{m}$; therefore, this structure is referred to as the equal width structure. The width, number of interdigitated pairs, effective impedance, and limiting current density for these three structures are listed in Table II. For the current density evaluation, the metal layers are extracted individually using FastHenry.

In the pyramid structure, the current density is maintained equal among the layers, lowering the limiting current density. Since the thickness decreases with lower metal layers, the lines 


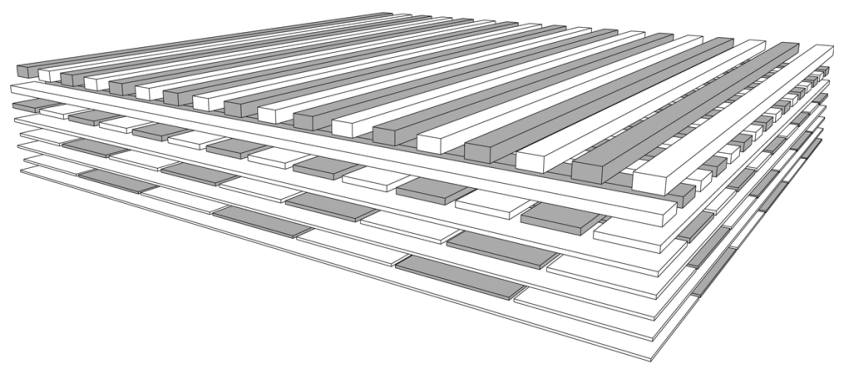

(a)

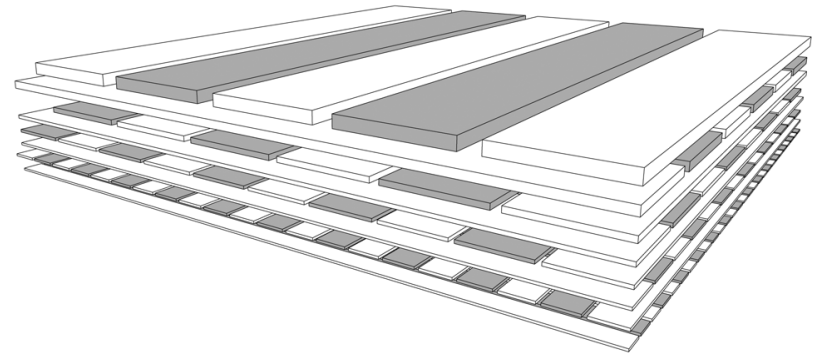

(b)

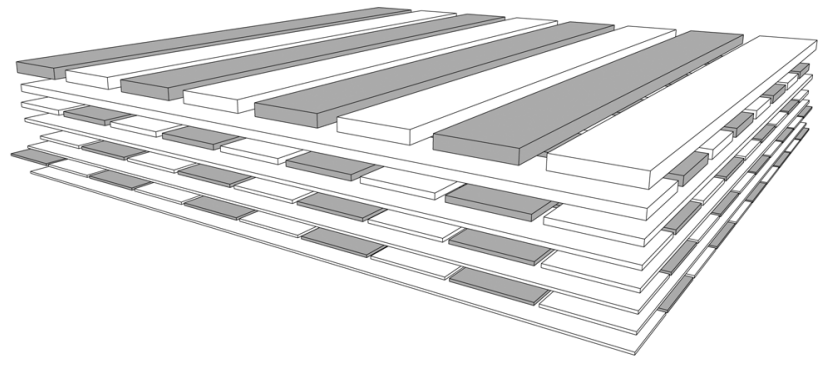

(c)

Fig. 16. Three P/G structures: (a) pyramid (proposed) structure-the width decreases with higher metal layers; (b) inverted pyramid (standard) structure- the width increases with higher metal layers; and (c) equal width structure-the width is maintained equal among all of the metal layers.

are wider, maintaining a constant current density. In the inverted pyramid structure, the higher metal layers are wider, permitting greater routing flexibility; the reliability of the metal, however, decreases since the limiting current density is $82 \%$ higher than the pyramid structure. In the inverted pyramid structure, most of the current flows in the higher metal layers increasing the effective impedance of the overall system. The impedance is $50 \%$ higher than in the pyramid structure. The equal width structure exhibits a higher effective impedance and current density of $24 \%$ and $36 \%$, respectively, as compared to the pyramid structure. This trend is consistent with the change in importance of the inductance as compared to the resistance at higher frequencies.

\section{B. Second Approach-Minimum Impedance}

The focus of the second optimization approach is to minimize the impedance of the overall $\mathrm{P} / \mathrm{G}$ distribution network. Assuming the metal layers are in parallel while optimizing each layer for minimum impedance, the lowest impedance of the overall system is achieved. The number of required metal layers is based on the current density constraint. Pseudo-code for this optimization algorithm is presented in Appendix C. The impedance of each of the eight metal layers is illustrated in Fig. 17.
Three different interdigitated $\mathrm{P} / \mathrm{G}$ distribution structures, illustrated in Fig. 16, are compared. The structures are referred to by the same names as in the previous subsection, however, the widths are determined based on the minimum impedance algorithm rather than the equal current density algorithm. The width of the power and ground lines for the pyramid (proposed) structure is based on the algorithm presented in Appendix C. In the inverted pyramid (standard) structure, the width increases with higher metal layers. The width of the metal layers is the opposite of the pyramid structure. The width of all eight metal layers is maintained constant at $2.4 \mu \mathrm{m}$ for the equal width structure. In Table III, the thickness, spacing, width, and number of interdigitated pairs are listed for each structure. The effective impedance and limiting current density for each structure are also summarized in Table III.

The lowest effective impedance is achieved in the pyramid structure. The effective impedance is $6 \%$ and $3 \%$ higher for the inverted pyramid and equal width structures, respectively, as compared to the pyramid structure. The limiting current density in the pyramid structure is enhanced, respectively, by $8 \%$ and $4 \%$ as compared to the inverted pyramid and equal width structures. Hence, the effective impedance achieved by the proposed pyramid structure is lower. This improvement is due to the relative importance of the inductance as compared to the resistance in high frequency systems.

\section{DISCUSSION}

The following section is divided into four subsections: a comparison between the two aforementioned design approaches, a discussion of routability and the grid area ratio, an estimate of the optimal $\mathrm{P} / \mathrm{G}$ line width for different frequencies and technologies, and an investigation of the critical frequency for the design of multi-layer $\mathrm{P} / \mathrm{G}$ networks.

\section{A. Comparison}

Evaluating both approaches, a tradeoff is observed between the impedance (or voltage drop) and the limiting current density of a $\mathrm{P} / \mathrm{G}$ distribution network. When focusing only on the current density, the optimal solution suggests the P/G network should be as wide as possible; however, at high frequencies, the effective impedance increases significantly due to the higher inductance. Both approaches consider the effective impedance and current density, while the current density is the focus of the first approach, and the effective impedance is the focus of the second approach. A comparison between both approaches for a one, two, three, and eight metal layer system is listed in Table IV.

As observed from Table IV, the effective impedance is lowest using the second approach, while the limiting current density is lowest if the first approach is used. A tradeoff between the limiting current density and effective impedance is noted.

A difference of less than $10 \%$ between the two approaches for the impedance and current density is demonstrated. However, when additional constraints (such as routability) are considered, the optimal width may not be available for that particular layer of metal within the $\mathrm{P} / \mathrm{G}$ distribution network. In this situation, the optimization process is focused on minimizing the impedance or current density, resulting in a greater difference 
TABLE II

Three Structures are Compared for Equal Current Density. The Thickness, Spacing, Width, and Number of InTerdigitated Pairs Per Metal LAYER FOR EACH STRUCTURE ARE LISTED

\begin{tabular}{|c|c|c||c|c||c|c||c|c|}
\hline \multirow{2}{*}{ Metal Layer } & \multirow{2}{*}{ Thickness $[\mu \mathrm{m}]$} & \multirow{2}{*}{ Spacing $[\mu \mathrm{m}]$} & \multicolumn{2}{c||}{ Pyramid structure } & \multicolumn{2}{|c||}{ Inverted pyramid structure } & \multicolumn{2}{|c|}{ Equal width structure } \\
\cline { 5 - 8 } & & & Width $[\mu \mathrm{m}]$ & $\begin{array}{c}\text { Number } \\
\text { of pairs }\end{array}$ & Width $[\mu \mathrm{m}]$ & $\begin{array}{c}\text { Number } \\
\text { of pairs }\end{array}$ & Width $[\mu \mathrm{m}]$ & $\begin{array}{c}\text { Number } \\
\text { of pairs }\end{array}$ \\
\hline \hline 8 & 0.975 & 0.540 & 1.7 & 227 & 9.0 & 52 & 5.5 & 82 \\
7 & 0.650 & 0.360 & 2.4 & 183 & 8.1 & 59 & 5.5 & 85 \\
6 & 0.430 & 0.240 & 3.6 & 131 & 7.7 & 63 & 5.5 & 87 \\
5 & 0.300 & 0.165 & 5.1 & 94 & 6.1 & 79 & 5.5 & 88 \\
4 & 0.250 & 0.140 & 6.1 & 79 & 5.1 & 95 & 5.5 & 88 \\
3 & 0.200 & 0.110 & 7.7 & 64 & 3.6 & 136 & 5.5 & 89 \\
2 & 0.190 & 0.105 & 8.1 & 61 & 2.4 & 202 & 5.5 & 89 \\
1 & 0.170 & 0.105 & 9.0 & 54 & 1.7 & 280 & 5.5 & 89 \\
\hline
\end{tabular}

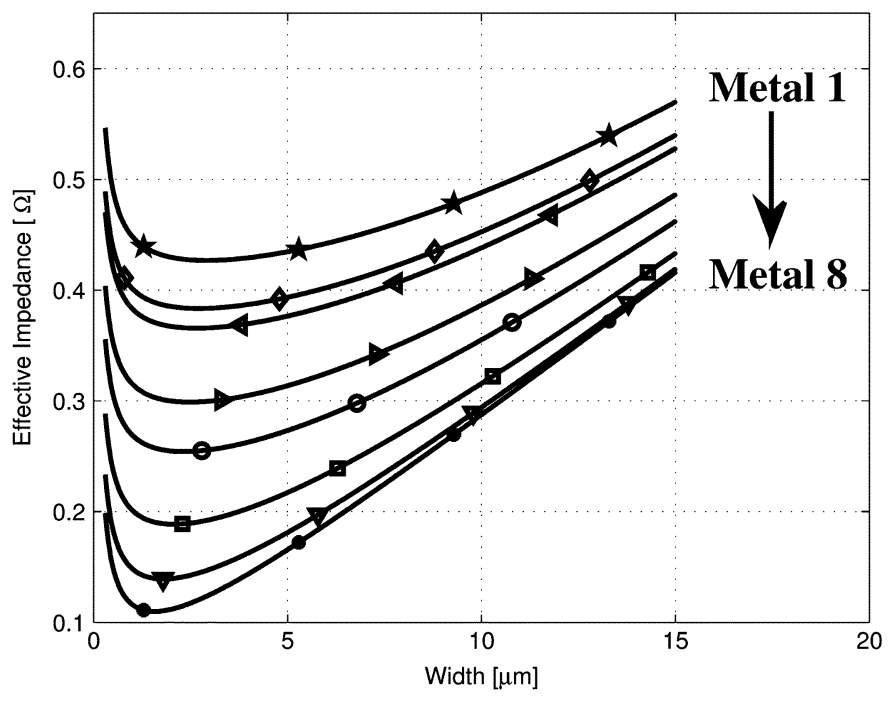

Fig. 17. Effective impedance as a function of width for each of eight metal layers within an interdigitated $\mathrm{P} / \mathrm{G}$ distribution network. The overall area of each metal layer is maintained constant.

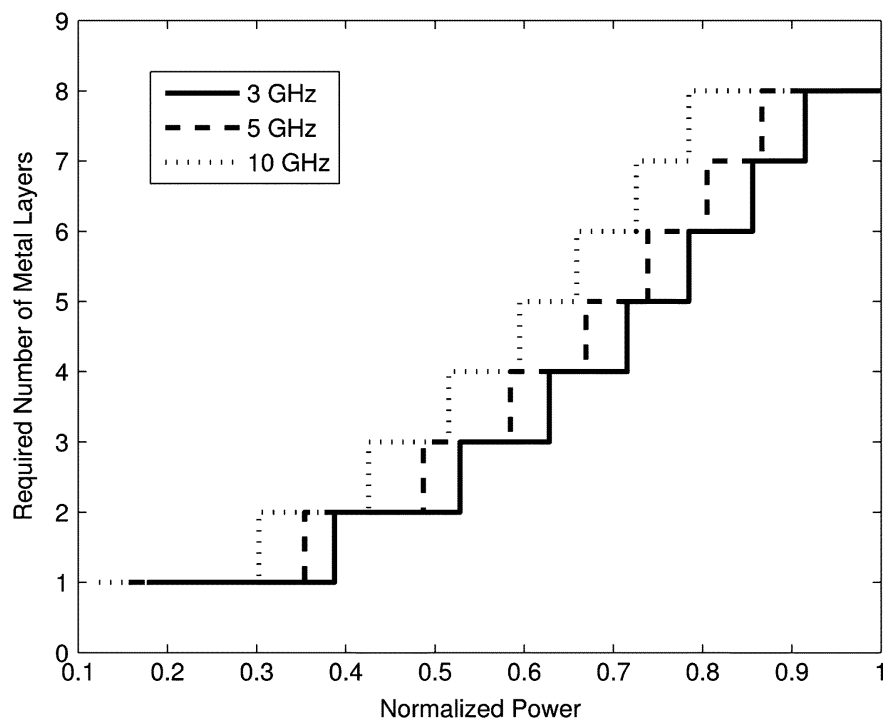

Fig. 18. Required number of metal layers for a $\mathrm{P} / \mathrm{G}$ network as a function of normalized power evaluated at three different frequencies.

between the two approaches. These two approaches are presented here to satisfy both optimization flows.

\section{B. Routability}

To develop the proposed methodology, these examples assume all of the metal layers and physical area can be used for the P/G network. For a practical on-chip power and ground distribution network, routability, cost, and other issues should also be considered. Routability is an important issue primarily affecting the lower metal layers. Global P/G networks tend to utilize the higher metal layers. To consider routability, a metric, the grid area ratio, is introduced as the ratio of the metal resources occupied by the P/G network to the total metal area [7], [18]

$$
A \equiv \frac{w+s_{0}}{p}
$$

where $w, s_{o}$, and $p$ are, respectively, the line width, minimal spacing between $\mathrm{P} / \mathrm{G}$ lines, and the line pitch. The line pitch is the width and spacing between the power and ground lines. If the spacing between the power and ground lines is minimum, the grid area ratio is one. The grid area ratio is depicted in Fig. 19 for different spacings. As anticipated, increasing the distance between the lines reduces the grid area ratio. As illustrated in Fig. 19, the grid area ratio is higher for the lower metal layers, resulting in reduced routability for the lower metal layers as compared to the higher metal layers where routability is better.

In Fig. 20, several $\mathrm{P} / \mathrm{G}$ networks with different line widths between the top and bottom metal layers are evaluated. A 5 $\mathrm{GHz}$ target frequency and $10 \mu \mathrm{m}$ line-to-line spacing between the power and ground lines is chosen. Four interdigitated metal layers are allocated for the power network. In Fig. 20, the $x$-axis is $w_{\text {top }} / w_{\text {bottom }}$, permitting a comparison between the impedance and grid-area-ratio for several pyramid, equal width, and inverted pyramid structures. The vertical line at $w_{\text {top }} / w_{\text {bottom }}=1$ represents the equal width structure. The region to the left of the equal width structure represents pyramid structures with increasing width at the bottom metal layers and decreasing width at the top layers. The region to the right represents inverted pyramid structures with decreasing width at the bottom metal layers and increasing width at the top layers. The lowest impedance among these structures is the pyramid structure with a line width based on (11). The grid-area-ratio however is lower in the inverted pyramid structure, indicating a tradeoff between the impedance and routability. The primary disadvantage of the proposed pyramid structure is therefore a higher grid-area-ratio (lower routability) as compared to the 
TABLE III

Three Structures are Compared for Minimum ImPedance. The ThickNess, Spacing, Width, and Number of Interdigitated Pairs Per Metal LAYER FOR EACH STRUCTURE ARE LISTED

\begin{tabular}{|c|c|c||c|c||c|c||c|c|}
\hline \multirow{2}{*}{ Metal Layer } & \multirow{2}{*}{ Thickness $[\mu \mathrm{m}]$} & \multirow{2}{*}{ Spacing $[\mu \mathrm{m}]$} & \multicolumn{2}{c||}{ Pyramid structure } & \multicolumn{2}{c||}{ Inverted pyramid structure } & \multicolumn{2}{|c|}{ Equal width structure } \\
\cline { 4 - 8 } & & & Width $[\mu \mathrm{m}]$ & $\begin{array}{c}\text { Number } \\
\text { of pairs }\end{array}$ & Width $[\mu \mathrm{m}]$ & $\begin{array}{c}\text { Number } \\
\text { of pairs }\end{array}$ & Width $[\mu \mathrm{m}]$ & $\begin{array}{c}\text { Number } \\
\text { of pairs }\end{array}$ \\
\hline \hline 8 & 0.975 & 0.540 & 1.7 & 227 & 2.9 & 144 & 2.4 & 170 \\
7 & 0.650 & 0.360 & 1.9 & 225 & 2.7 & 162 & 2.4 & 181 \\
6 & 0.430 & 0.240 & 2.1 & 212 & 2.7 & 172 & 2.4 & 189 \\
5 & 0.300 & 0.165 & 2.3 & 199 & 2.5 & 187 & 2.4 & 194 \\
4 & 0.250 & 0.140 & 2.5 & 189 & 2.3 & 201 & 2.4 & 196 \\
3 & 0.200 & 0.110 & 2.7 & 180 & 2.1 & 225 & 2.4 & 199 \\
2 & 0.190 & 0.105 & 2.7 & 177 & 1.9 & 254 & 2.4 & 199 \\
1 & 0.170 & 0.105 & 2.9 & 165 & 1.7 & 283 & 2.4 & 199 \\
\hline
\end{tabular}

TABLE IV

COMPARISON BETWEEN TWO OPTIMIZATION APPROACHES FOR A ONE, TWO, Three, and Eight Metal LaYer System. CD IS THE CuRrent Density

\begin{tabular}{|c||c|c||c|c|}
\hline \multicolumn{1}{|c||}{$\begin{array}{c}\text { Number } \\
\text { of metal } \\
\text { layers }\end{array}$} & \multicolumn{2}{c||}{ First Approach } & \multicolumn{2}{c|}{ Second Approach } \\
\cline { 2 - 5 } & $Z_{e f f}[\mathrm{~m} \Omega]$ & $\begin{array}{c}\text { Limiting CD } \\
{\left[\mathrm{mA} / \mu \mathrm{m}^{2}\right]}\end{array}$ & $Z_{e f f}[\mathrm{~m} \Omega]$ & $\begin{array}{c}\text { Limiting CD } \\
{\left[\mathrm{mA} / \mu \mathrm{m}^{2}\right]}\end{array}$ \\
\hline \hline 1 & 105.1 & 2.71 & 105.1 & 2.71 \\
2 & 59.5 & 1.54 & 59.4 & 1.60 \\
3 & 45.6 & 1.18 & 45.2 & 1.25 \\
8 & 30.6 & 0.77 & 29.5 & 0.84 \\
\hline
\end{tabular}

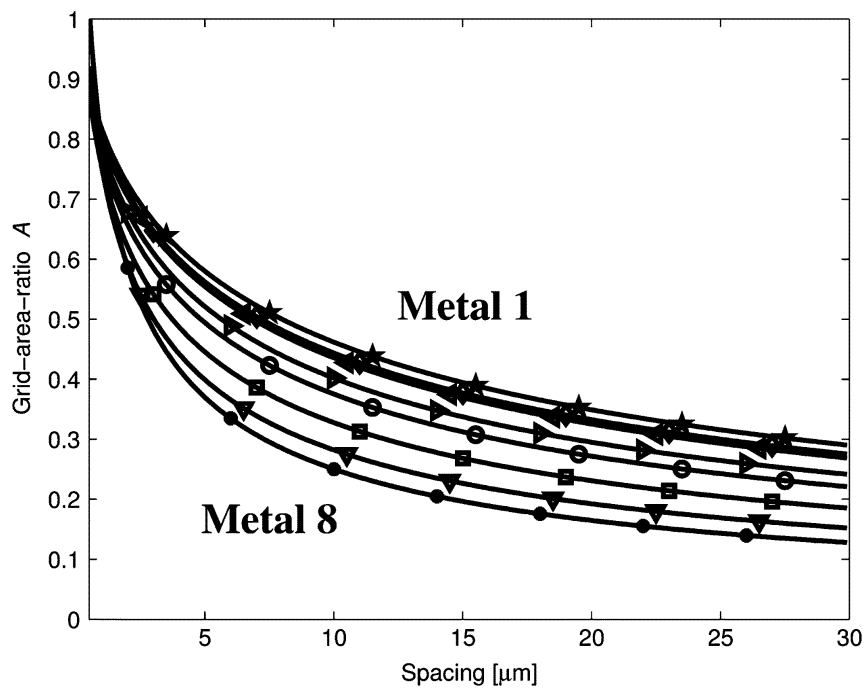

Fig. 19. Grid area ratio as a function of spacing between the lines for different metal layers. The line width is based on (11) to minimize the network impedance.

conventional inverted pyramid structure. A power network to the right of the minimum impedance pyramid structure may therefore be a reasonable compromise to provide effective routability while tolerating a reasonable increase in network impedance.

\section{Fidelity}

The required number of metal layers for the specified power levels is depicted in Fig. 18. The technology parameters are chosen based on a 65-nm CMOS technology with an area of $1 \mathrm{~mm} \times 1 \mathrm{~mm}$. The results are evaluated at three different frequencies, indicating that an additional metal layer is required at higher frequencies.

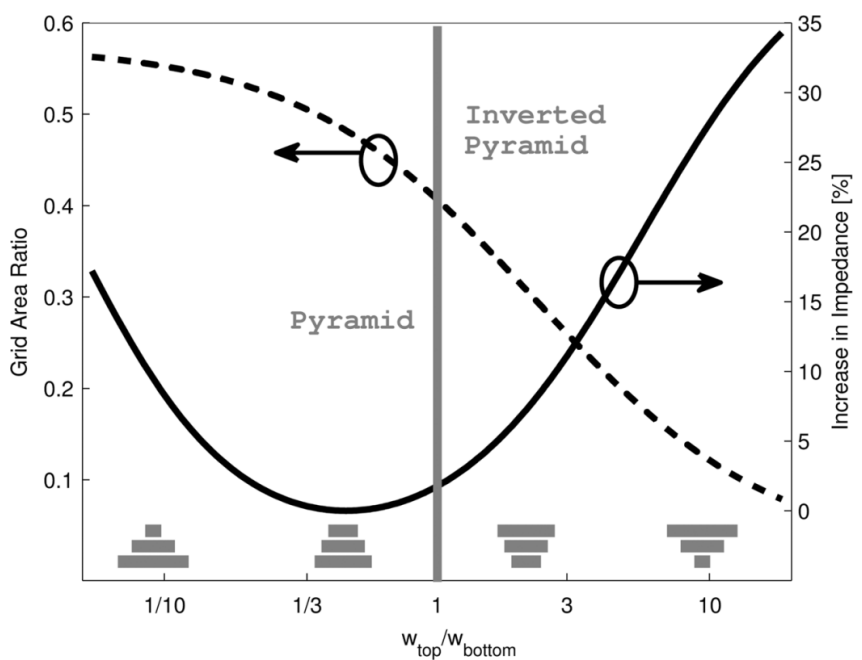

Fig. 20. Grid-area-ratio and increase in impedance for several interdigitated P/G structures. Four metal layers are allocated for the power network. The vertical line represents the equal width structure. The left region is for pyramid structures, while the right region is for inverted pyramid structures. The minimum impedance is achieved by the pyramid structure, where the line width is based on (11).

The optimal width as a function of the number of metal layers at 3 and $10 \mathrm{GHz}$ is illustrated in Fig. 21 for a 65-, 45- [24], and 32-nm [25] CMOS technology. The optimal width is determined baaed on the Newton-Raphson method as described by (11). At higher frequencies, the optimal width is thinner since the inductive impedance is greater. The optimal width increases with thinner, less inductive metal layers to satisfy the minimum impedance constraint. With technology scaling, metal thicknesses typically decrease, requiring wider lines to compensate for the increase in resistivity.

The effect of frequency on the design of an interdigitated P/G distribution network is significant. At lower frequencies, where the resistive impedance is dominant, wide wires are used to reduce the impedance, while maintaining a constant cross-section to satisfy equal current density. At higher frequencies, the inductive impedance is dominant, suggesting that the $\mathrm{P} / \mathrm{G}$ lines should be less wide.

\section{Critical Frequency}

The relationship between the two highest metal layers is investigated, permitting the concept of a critical frequency to be defined. The critical frequency is described here as the 


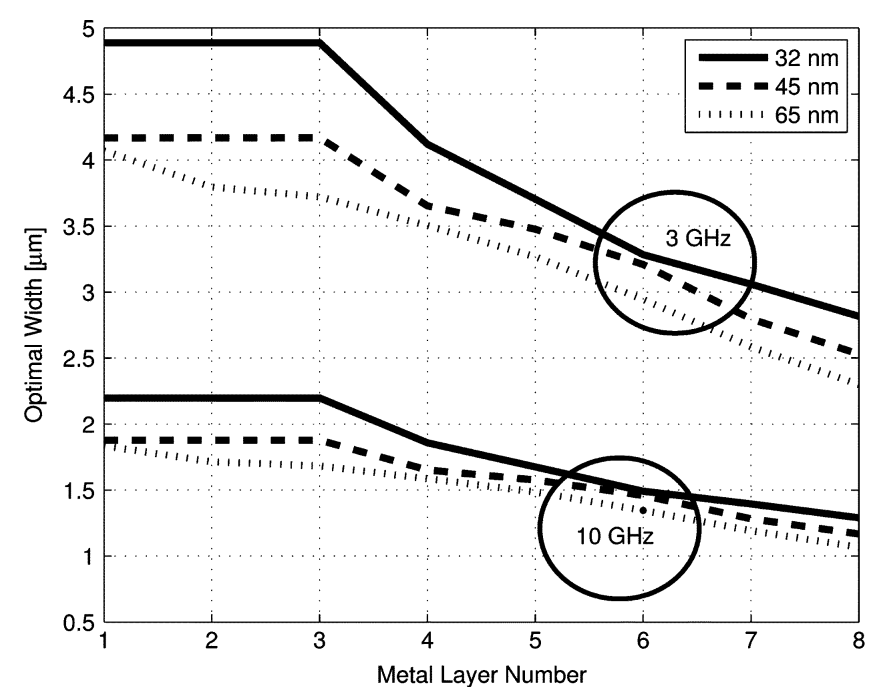

Fig. 21. Optimal width to minimize the effective impedance of each metal layer based on a 65-, 45-, and 32-nm CMOS technology for two different frequencies.

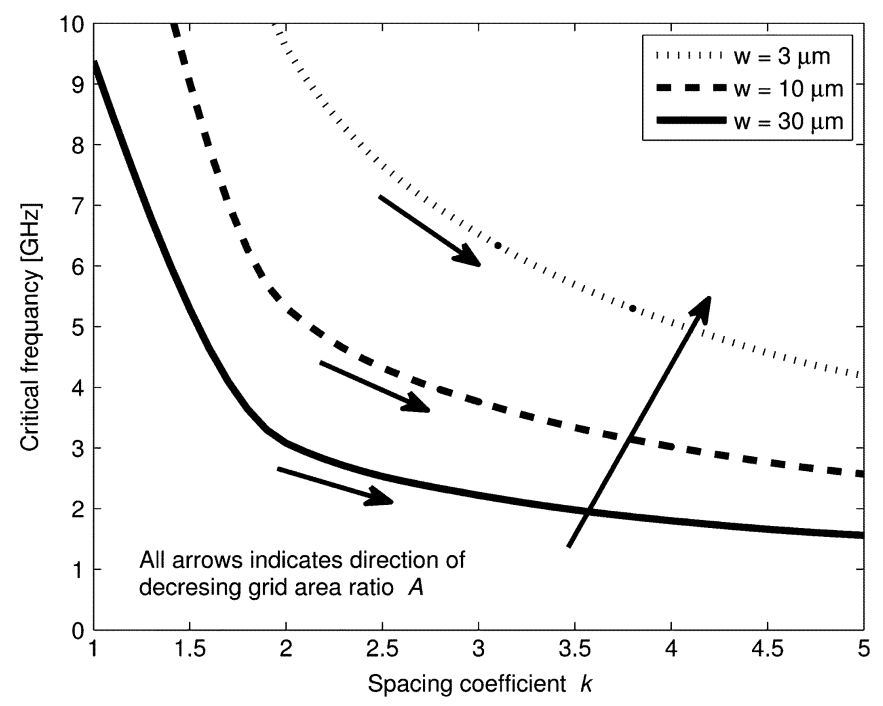

Fig. 22. Critical frequency at which the impedance of the higher metal layer is equal to the impedance of the lower metal layer. The width of the P/G lines is maintained equal for both metal layers. The distance between the lines is the minimum spacing of each layer multiplied by a spacing coefficient $k$.

frequency at which the impedance of two metal layers is equal. Assuming the width of both metal layers is the same, the critical frequency is determined for a variety of spacings, as depicted in Fig. 22. The arrows indicate the direction of decreasing grid area ratio $A$ (increasing routability). The critical frequency is evaluated for three different line widths.

The critical frequency for different grid area ratios is illustrated in Fig. 23. A line width of $10 \mu \mathrm{m}$ is assumed. The area above the target frequency is the region where the higher metal layer is dominant (the impedance of the higher metal layer is greater), while the area below the target frequency is the region where the lower metal layer is dominant (the impedance of the lower layer is greater). From Fig. 23, the higher metal layer is the dominant metal layer in terms of the impedance for signal frequencies above 3 (for high routability) and $16 \mathrm{GHz}$ (for low routability), assuming a $10 \mu \mathrm{m}$ line width for both metal layers.

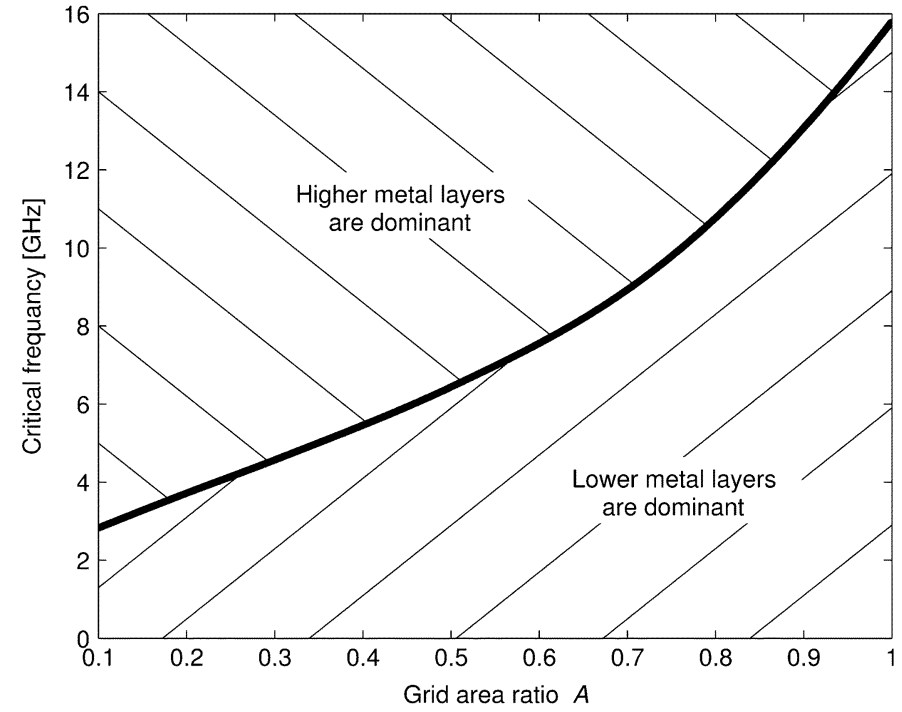

Fig. 23. Critical frequency as a function of grid area ratio. A line width of 10 $\mu \mathrm{m}$ is assumed for all $\mathrm{P} / \mathrm{G}$ lines. The area above the line indicates the region where the higher metal layer is dominant, while the region below the line indicates the region where the lower metal layer is dominant.

\section{CONCLUSION}

The characteristics of inductance (including mutual) in interdigitated multi-layer $\mathrm{P} / \mathrm{G}$ distribution network are investigated in this paper. A closed-form expression for the inductance of a single metal layer within an interdigitated $P / G$ network structure is presented. The model is compared with FastHenry and exhibits high accuracy and low complexity, permitting efficient estimation of the inductance in an interdigitated $\mathrm{P} / \mathrm{G}$ network.

Based on this analytic model, the impedance of a single layer within an interdigitated $\mathrm{P} / \mathrm{G}$ network structure is minimized, permitting an efficient and accurate estimate of the optimal width of the power and ground wires. This result has been favorably compared to FastHenry. For 65-, 45-, and 32-nm CMOS technologies, the optimal width of each metal layer for minimum effective impedance is determined. The effect of the physical dimensions of the metal and the signal frequency on the optimal width is also discussed.

Considering the current density, two approaches for designing a multi-layer interdigitated $\mathrm{P} / \mathrm{G}$ distribution network are evaluated. For each approach, the pyramid (proposed), inverted pyramid (standard), and equal width $\mathrm{P} / \mathrm{G}$ structure are considered. The proposed methodology for equal current density improves the effective impedance and limiting current density by $50 \%$ and $82 \%$, respectively, as compared with the conventional inverted pyramid structure. The methodology for the minimum impedance achieves a $6 \%$ and $8 \%$ enhancement, respectively, as compared to the inverted pyramid structure for the effective impedance and current density. This behavior is due to the relative change in importance of the inductance as compared to the resistance in high frequency systems.

The grid area ratio is introduced, demonstrating enhanced routability in the higher metal layers as compared with the lower metal layers. Under different routability constraints, the frequency at which the higher metal layers are more dominant than the lower metal layers is determined. Several pyramid, 


\section{EQUAL-CURRENT-DENSITY}

1. Optimize the top metal layer width, based on (10) and (11).

2. Determine $R_{1}, L_{1}$, and $Z_{1}$.

3. Determine the current density for a single layer, $n=1$.

4. while (allowed maximum current density $<$ limiting current density)

Increase a number of metal layers, $n=n+1$.

Determine widt $_{n}$, based on (19).

Determine $R_{n}, L_{n}$, and $Z_{n}$.

Determine the current density for each layer.

9. end

Fig. 24. Pseudo-code for the first optimization approach. The widths are chosen to maintain equal current density among each of the layers.

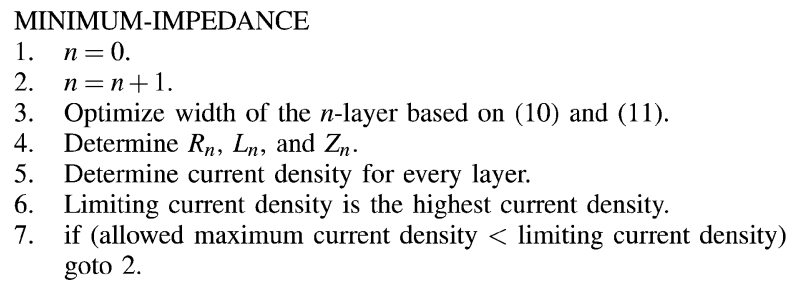

Fig. 25. Pseudo-code for the second optimization approach. The widths are determined to achieve the minimum impedance for each individual metal layer.

equal width, and inverted pyramid structures are compared in terms of the impedance and grid-area-ratio, indicating a tradeoff between the impedance and routability.

\section{APPENDIX A}

\section{ESTIMATE OF INITIAL OPTIMAL WIDTH}

Since the effective inductance is a transcendental function of width, no closed-form analytic solution can be determined for the wire width that minimizes the effective impedance. The line thickness $t$ is replaced with $t_{\text {ind }}$ to simplify the effective inductance model when determining the optimal width. The effective inductance for an interdigitated structure where the distance between the power and ground wires is equal to the thickness of the metal is

$$
\left\langle L_{\mathrm{eff}}\right\rangle_{s=t_{\mathrm{ind}}}=\frac{2 l(w+s)}{A} \frac{\mu_{0} l}{\pi}\left[\frac{3}{2}+\ln \left(\frac{2}{\pi}\right)\right] .
$$

The minimum impedance is determined by solving for the root of the derivative of $\left|Z_{\text {eff }}(w)\right|_{s=t_{\text {ind }}}$

$$
\frac{\partial\left|\left[Z_{\mathrm{eff}}(w)\right]_{s=t_{\text {ind }}}\right|}{\partial w}=0 .
$$

A closed-form solution for the wire width that produces the minimum impedance assuming $s=t_{\text {ind }}$ is

$$
w_{\mathrm{opt}}=\left(\frac{1}{\left[\frac{3}{2}+\ln \left(\frac{2}{\pi}\right)\right]^{2}}\right)^{1 / 3} \sqrt[3]{\frac{s \rho^{2}}{\mu_{o}^{2} t^{2} f^{2}}} .
$$

\section{APPENDIX B \\ FIRST OPTIMIZATION APPROACH}

The input to the EQUAL-CURRENT-DENSITY algorithm, illustrated in Fig. 24, is the technology parameters for each metal layer in the system, the physical dimensions, and the total current. At line 1, the width of the top metal layer is determined. The process is initiated from the top metal layer since this layer is thickest, permitting a solution for the width of the remaining metal layers. If the current density determined in line 3 is greater than the maximum current density allowed by the technology, additional metal layers should be allocated for the $\mathrm{P} / \mathrm{G}$ distribution network. The width of the additional metal layers is determined from (19) to lower the limiting current density within the $\mathrm{P} / \mathrm{G}$ network. At higher frequencies, the skin depth is considered when evaluating the current density. $n$ represents the minimum number of metal layers required to effectively distribute power and ground.

\section{APPENDIX C SECOND OPTIMIZATION APPROACH}

The MINIMUM-IMPEDANCE pseudo-code, presented in Fig. 25, is based on minimizing the impedance of each metal layer within a multi-layer $\mathrm{P} / \mathrm{G}$ system. Note the optimization algorithm begins from the highest metal layer and decreases as required. A specific metal width is determined in line 3 . In line 4 , the impedance of the current metal layer is determined. The current density is recalculated for each metal layer in line 5. If the maximum current density allowed by the technology is lower than the limiting current density, the algorithm returns to line 2, assigning an additional metal layer for the $\mathrm{P} / \mathrm{G}$ structure. $n$ represents the minimum number of metal layers required for the $\mathrm{P} / \mathrm{G}$ network.

\section{REFERENCES}

[1] H. B. Bakoglu, Circuits, Interconnects, and Packaging for VLSI. Reading, MA: Addison-Wesley, 1990.

[2] K. T. Tang and E. G. Friedman, "Incorporating voltage fluctuations of the power distribution network into the transient analysis of CMOS logic gates," Analog Integr. Circuits Signal Process., vol. 31, no. 3, pp. 249-259, Jun. 2002.

[3] A. H. Ajami, K. Banerjee, A. Mehrotra, and M. Pedram, "Analysis of IR-drop scaling with implications for deep submicron P/G network designs," in Proc. IEEE Int. Symp. Quality Electron. Des., Mar. 2003, pp. 35-40.

[4] K. T. Tang and E. G. Friedman, "Simultaneous switching noise in on-chip CMOS power distribution networks," IEEE Trans. Very Large Scale Integr. (VLSI) Syst., vol. 10, no. 4, pp. 487-493, Aug. 2002.

[5] D. A. Priore, "Inductance on silicon for sub-micron CMOS VLSI," in Proc. IEEE Symp. VLSI Circuits, May 1993, pp. 17-18.

[6] L.-R. Zheng and H. Tenhunen, "Effective power and ground distribution scheme for deep submicron high speed VLSI circuits," in Proc. IEEE Int. Symp. Circuits Syst., May 1999, vol. I, pp. 537-540.

[7] M. Popovich, A. V. Mezhiba, and E. G. Friedman, Power Distribution Networks With On-Chip Decoupling Capacitors. New York: Springer, 2008.

[8] A. V. Mezhiba and E. G. Friedman, "Inductance properties of highperformance power distribution grids," IEEE Trans. Very Large Scale Integr. (VLSI) Syst., vol. 10, no. 6, pp. 762-776, Dec. 2002.

[9] International Technology Roadmap for Semiconductors 2007. [Online]. Available: http://public.itrs.net 
[10] W. S. Song and L. A. Glasser, "Power distribution techniques for VLSI circuits," IEEE J. Solid-State Circuits, vol. 21, no. 1, pp. 150-156, Feb. 1986.

[11] K.-H. Erhard, F. M. Johannes, and R. Dachauer, "Topology optimization techniques for power/ground networks in VLSI," in Proc. Eur. Des. Autom. Conf., Sep. 1992, pp. 362-367.

[12] J. Fu, X. Wu, X. Hong, and Y. Cai, "PG2000: A CAD tool for power/ ground network design, optimization and verification based on standard cell VLSIs," in Proc. IEEE Int. Conf. Commun., Circuits Syst., Jun. 2002, vol. 2, pp. 1424-1428.

[13] S. X. D. Tan, C. J. R. Shi, and J.-C. Lee, "Reliability-constrained area optimization of VLSI power/ground networks via sequence of linear programmings," IEEE Trans. Comput.-Aided Des. Integr. Circuits Syst., vol. 22, no. 12, pp. 1678-1684, Dec. 2003.

[14] K. Wang and M. Marek-Sadowska, "On-chip power-supply network optimization using multigrid-based technique," IEEE Trans. Comput.Aided Des. Integr. Circuits Syst., vol. 24, no. 3, pp. 407-417, Mar. 2005.

[15] J. Singh and S. S. Sapatnekar, "Partition-based algorithm for power grid design using locality," IEEE Trans. Comput.-Aided Des. Integr. Circuits Syst., vol. 25, no. 4, pp. 664-677, Apr. 2006.

[16] A. V. Mezhiba and E. G. Friedman, "Scaling trends of on-chip power distribution noise," IEEE Trans. Very Large Scale Integr. (VLSI) Syst., vol. 12, no. 4, pp. 386-394, Apr. 2004.

[17] D. Khalil and Y. Ismail, "Approximate frequency response models for RLC power grids," in Proc. IEEE Int. Symp. Circuits Syst., May 2007, pp. 3784-3787.

[18] N. Srivastava, X. Qi, and K. Banerjee, "Impact of on-chip inductance on power distribution network design for nanometer scale integrated circuits," in Proc. IEEE Int. Symp. Quality Electron. Des., Mar. 2005, pp. 346-351.

[19] R. Jakushokas and E. G. Friedman, "Inductance model of interdigitated power and ground distribution networks," IEEE Trans. Circuits Syst. II, Analog Digit. Process., vol. 56, no. 7, pp. 585-589, Jul. 2009.

[20] F. Grover, Inductance Calculation: Working Formulas and Tables. New York: Dover, 1962.

[21] J. Wallis, Opera Mathematica. Leon: Lichfield Academiæ Typographi, 1656.

[22] M. Kamon, M. J. Tsuk, and J. K. White, "FastHenry: A multipoleaccelerated 3-D inductance extraction program," IEEE Trans. Microw. Theory Tech., vol. 42, no. 9, pp. 1750-1758, Sep. 1994.

[23] P. Bai, C. Auth, S. Balakrishnan, M. Bost, R. Brain, V. Chikarmane, R. Heussner, M. Hussein, J. Hwang, D. Ingerly, R. James, I. Jeong, C. Kenyan, E. Lee, S.-H. Lee, N. Lindert, M. Liu, Z. Ma, T. Marieb, A. Murthy, R. Nagisetty, S. Natarajan, J. Neirynck, A. Ott, C. Parker, J. Sebastian, R. Shaheed, S. Sivakumar, J. Steigenvald, S. Tyagi, C. Weber, B. Woolely, A. Yeoh, K. Zhang, and M. Bohr, "A 65 nm logic technology featuring $35 \mathrm{~nm}$ gate lengths, enhanced channel strain, 8 $\mathrm{Cu}$ interconnect layers, low-k ILD and $0.57 \mu \mathrm{m}^{2}$ SRAM cell," in Proc. IEEE Int. Electron Devices Meet., Dec. 2004, pp. 657-660.

[24] K. Mistry, C. Allen, C. Auth, B. Beattie, D. Bergstrom, M. Bost, M. Brazier, M. Buehler, A. Cappellani, R. Chau, C.-H. Choi, G. Ding, K. Fischer, T. Ghani, R. Grover, W. Han, D. Hanken, M. Hattendorf, J. He, J. Hicks, R. Huessner, D. Ingerly, P. Jain, R. James, L. Jong, S. Joshi, C. Kenyon, K. Kuhn, K. Lee, H. Liu, J. Maiz, B. McIntyre, P. Moon, J. Neirynck, S. Pae, C. Parker, D. Parsons, C. Prasad, L. Pipes, M. Prince, P. Ranade, T. Reynolds, J. Sandford, L. Shifren, J. Sebastian, J. Seiple, D. Simon, S. Sivakumar, P. Smith, C. Thomas, T. Troeger, P. Vandervoorn, S. Williams, and K. Zawadzki, "A $45 \mathrm{~nm}$ logic technology with high- $\mathrm{k}+$ metal gate transistors, strained silicon, $9 \mathrm{Cu}$ interconnect layers, $193 \mathrm{~nm}$ dry patterning, and 100\% Pb-free packaging," in Proc. IEEE Int. Electron Devices Meet., Dec. 2007, pp. 247-250.
[25] S. Natarajan, M. Armstrong, M. Bost, R. Brain, M. Brazier, C.-H. Chang, V. Chikarmane, M. Childs, H. Deshpande, K. Dev, G. Ding, T. Ghani, O. Golonzka, W. Han, J. He, R. Heussner, R. James, I. Jin, C. Kenyon, S. Klopcic, S.-H. Lee, M. Liu, S. Lodha, B. McFadden, A. Murthy, L. Neiberg, J. Neirynck, P. Packan, S. Pae, C. Parker, C. Pelto, L. Pipes, J. Sebastian, J. Seiple, B. Sell, S. Sivakumar, B. Song, K. Tone, T. Troeger, C. Weber, M. Yang, A. Yeoh, and K. Zhang, "A $32 \mathrm{~nm}$ logic technology featuring $2 \mathrm{nd}$-generation high- $\mathrm{k}+$ metal gate transistors, enhanced channel strain and $0.171 \mu \mathrm{m}^{2}$ SRAM cell size in a $291 \mathrm{Mb}$ array," in Proc. IEEE Int. Electron Devices Meet., Dec. 2008, pp. 1-3.

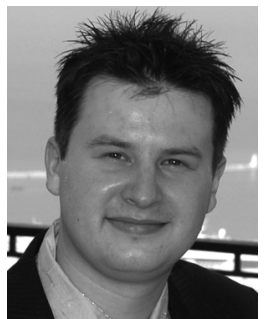

Renatas Jakushokas (S'09) received the B.Sc. degree in electrical engineering from Ort-Braude College, Karmiel, Israel, in 2005, and the M.S. degree in electrical and computer engineering from the University of Rochester, Rochester, NY, in 2007, where he is currently pursuing the $\mathrm{Ph} . \mathrm{D}$. degree in electrical and computer engineering.

He was previously an intern with Intrinsix Corporation, Fairport, NY, in 2006, working on Sigma Delta ADCs, Eastman Kodak Company, Rochester, NY, in 2007, working on high performance comparators, and Freescale Semiconductor Corporation, Tempe, AZ, in 2008, where he worked on evaluating substrate isolation techniques. His research interests include the areas of power distribution, noise, signal integrity, and optimization techniques in high performance integrated circuit design methodologies.

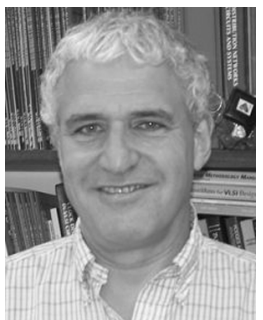

Eby G. Friedman (S'78-M'79-SM'90-F'00) received the B.S. degree from Lafayette College in 1979, and the M.S. and Ph.D. degrees from the University of California, Irvine, in 1981 and 1989, respectively, all in electrical engineering.

From 1979 to 1991, he was with Hughes Aircraft Company, rising to the position of Manager of the Signal Processing Design and Test Department, responsible for the design and test of high performance digital and analog IC's. He has been with the Department of Electrical and Computer Engineering, University of Rochester, Rochester, NY, since 1991, where he is a Distinguished Professor. He is also a Visiting Professor with the Technion-Israel Institute of Technology. His current research and teaching interests include high performance synchronous digital and mixed-signal microelectronic design and analysis with application to high speed portable processors and low power wireless communications. He is the author of about 350 papers and book chapters, several patents, and the author or editor of 12 books in the fields of high speed and low power CMOS design techniques, high speed interconnect, and the theory and application of synchronous clock and power distribution networks.

Dr. Friedman is the Regional Editor of the Journal of Circuits, Systems and Computers, Chair of the IEEE TRAnsactions ON Very LaRge SCAlE INTEGRATION (VLSI) SYSTEMS steering committee, and a Member of several editorial boards and conference technical program committees. He previously was the Editor-in-Chief of the IEEE TRANSACTIONS ON VERY LARGE SCALE INTEGRATION (VLSI) SYSTEMS, a Member of the editorial board of the PROCEEDINGS OF THE IEEE, a Member of the Circuits and Systems (CAS) Society Board of Governors, Program and Technical chair of several IEEE conferences, and a recipient of the University of Rochester Graduate Teaching Award, and the College of Engineering Teaching Excellence Award. He is a Senior Fulbright Fellow. 